\title{
Ultrashort Pulse Laser Cutting of Intraocular Lens Polymers
}

\author{
Johannes HEBERLE $^{{ }^{1}, 2}$, Florian KLÄMPFL ${ }^{* 1}$, Ilya ALEXEEV ${ }^{* 1,2}$ and Michael SCHMIDT ${ }^{* 1,2}$ \\ ${ }^{* 1}$ Institute of Photonic Technologies, Friedrich-Alexander-Universität Erlangen-Nürnberg, \\ Paul-Gordan-Straße 3, 91052 Erlangen, Germany \\ E-mail: johannes.heberle@lpt.uni-erlangen.de \\ *2 School of Advanced Optical Technologies, Friedrich-Alexander-Universität Erlangen-Nürnberg, \\ Paul-Gordan-Straße 6, 91052 Erlangen, Germany
}

\begin{abstract}
Material processing by ultra-short pulsed laser ablation is an attractive approach for various applications. In this paper we present our studies on cutting of medical polymers used for intraocular lenses (IOL). To achieve best results the ablation characteristics and the ablation thresholds are identified for single and multiple irradiation of the lens material by picosecond pulses. The results are used for quantification of the incubation effect. Furthermore, cutting experiments are carried out and thereby different damage types can be observed. A process window is developed containing the regions of the respective types of damages and backed up by theoretical considerations.
\end{abstract}

DOI: $10.2961 /$ jlmn.2014.02.0005

Keywords: picosecond, ultrafast laser, ultrashort pulsed, near infrared, ablation, intraocular lens, cutting, incubation effect

\section{Introduction}

Various diseases of the human eye are treated in ophthalmology by the implantation of intraocular lenses (IOL). These lenses can either replace the natural eye lens as in cataract surgeries or they can be implanted in addition to the natural human lens to treat hyperopia, myopia or astigmatism if a refractive surgery (e.g. LASIK) is not possible. Intraocular lenses are made of conventional PMMA or modern hydrophobic or hydrophilic acrylate polymers [1]. An intraocular lens possesses two main functional parts: the centrally arranged refractive area used for imaging and the outer parts of the lens which are the haptics. The haptics enable fixation of the lens in different parts of the eye. Various designs of the haptics have been developed to react on the given physiological conditions of different kinds of tissues and to achieve strong stability (Fig. 1) preventing the lens from displacement inside the eye.

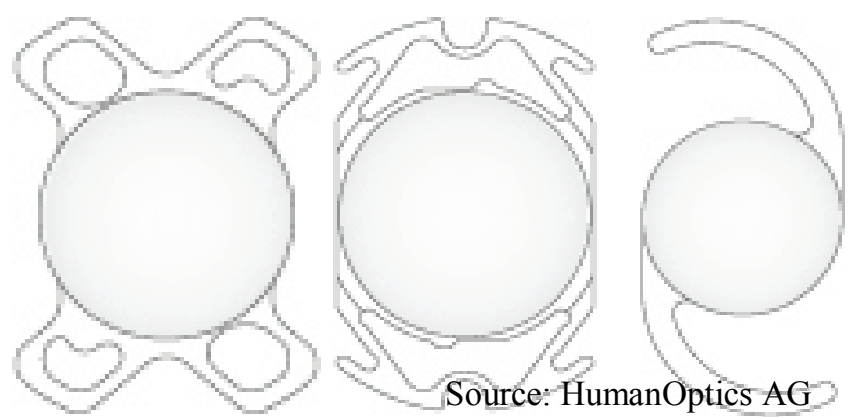

Fig. 1: Examples of intraocular lenses

Due to the high brittleness of these polymers and the high precision needed for manufacturing of such lenses, conventionally this is carried out by diamond tools, such as diamond turning of the refractive surface and diamond milling of the lens contour. Especially, production of the lens contour involves some problems. Tool wear causes deteriorating results at the lifetime end of such tools. The processing time is high due to the slow feed rates needed for controlled material removal. Finally, the minimal width of the produced kerfs is limited as they have at least the same size as the applied tool. Such tools have minimal diameters of about $\mathrm{d}=0.3 \mathrm{~mm}$ thus the flexibility of possible haptics designs is restricted.

Another thinkable production tool which may offer faster processing is the $\mathrm{CO}_{2}$-laser which has found many applications at cutting polymers. However, main disadvantages are the minimal achievable kerf width and large heat affected zones. In PMMA kerf widths on the order of several hundred micrometers are reported [10]. Since the laser beam is absorbed linearly and heat conduction into the surrounding material is present a comparably large area is molten and solidified again. These temperature changes could impair the functionality of the fragile haptics.

An alternative fabrication method which we propose in this paper is the use of ultrafast laser material ablation for the production of the contour. This approach is very capable and has gained significant attention in the last few years. Various applications of ultrashort laser pulses especially for medical applications such as stent production or shaping biomedical polymers have been established [3][4]. Also experiments on processing of intraocular lenses with ultrashort laser pulses have been carried out to achieve material modification and patterning of micro structures onto the lens surface.

The most promising laser system for IOL material removal is a high power picosecond laser since it delivers the required pulse energy levels and is suitable for industrial environment. To establish a manufacturing process, investigations on the laser ablation behavior of intraocular lens materials have to be performed. In the following section we present our results quantifying the incubation effect and identifying possible damage types at near infrared laser light. The use of frequency-tripled UV-light would also be possible as proposed for other applications for polymers; 
however, this is ruled out due the high linear absorption of the material. Due to this we expect significantly higher processing speeds possible and smaller heat affected zones than for ablation by linear absorption. Furthermore, suitable laser parameters are evaluated considering best quality and highest productivity.

\section{Experimental setup}

The experimental setup used for our studies is shown in Fig 2. The applied laser system is a mode locked Nd:YVO DPSS-laser (Fuego, Time-Bandwidth AG) with a wavelength $\lambda=1064 \mathrm{~nm}$, a pulse duration $\tau_{\mathrm{p}}=10 \mathrm{ps}$, a maximum mean power $P=35 \mathrm{~W}$ and a pulse frequency continuously variable between a single pulse and $f_{p}=8.2 \mathrm{MHz}$. The beam is expanded twice to a beam diameter of $2 w=6.7 \mathrm{~mm}$ and guided by a scanner. The scanner (Hurryscan, Scanlab AG) deflects the beam with two rotatable mirrors before it gets focused by an F-theta lens with a focal length $f=160 \mathrm{~mm}$ resulting in a focal spot diameter $2 w_{0}=31.5 \mu \mathrm{m}$ and a Rayleigh length $\mathrm{z}_{\mathrm{R}}=670 \mu \mathrm{m}$. The beam focus can be moved laterally in the focal plane of the lens with a maximum speed of $\mathrm{v}=10 \mathrm{~m} / \mathrm{s}$.

The samples are placed centrally below the scanner on a hollow spike in a hollow sample holder. Low pressure inside the spike prevents the lens from moving during the cutting procedure and the contour of the outer part of the sample holder supports the remaining negative after cutting. Due to this arrangement the transmitted parts of the incident laser beam are absorbed far enough from the sample to be defocussed enough to avoid ablation of the sample holder by high intensities.

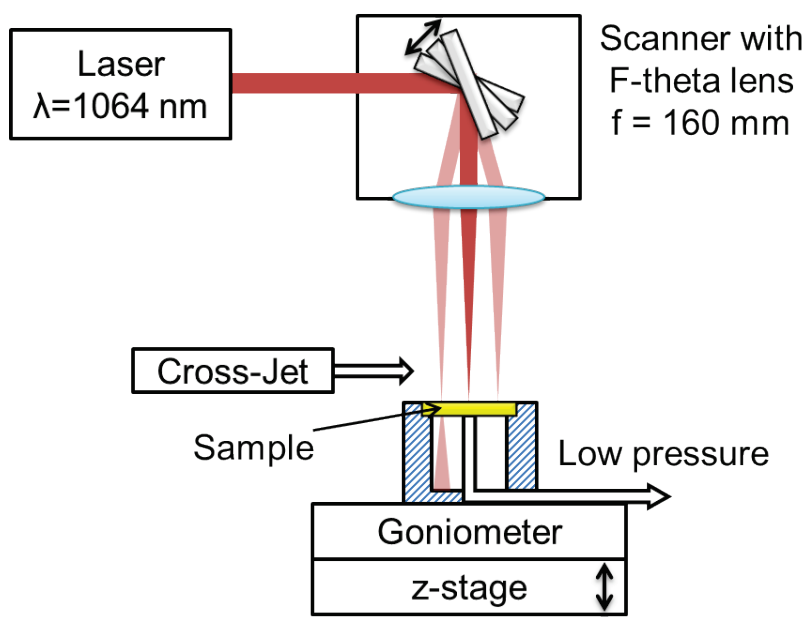

Fig. 2: Experimental setup for cutting medical polymer with a 10 ps laser.

The sample surface is positioned by a manual z-stage in the focal plane of the lens. An air flow with pressure $p=2$ bar above the sample, the cross-jet, prevents redeposition of already ablated debris on the sample. The ablated volume and the resulting surface roughness were measured with a laser scanning microscope (Olympus OLS 4000) to determine process efficiency and quality of the produced cutting kerfs.

The IOL material (Contamac Ltd.) [11] to be investigated is a commercially available copolymer of PHEMA (80 \%) and PMMA (19\%) with an absorber (1\%) for UV-light with the transmission spectrum shown in Fig. 3 measured by a spectrometer (Agilent Cary 5000 UV-Vis-NIR).

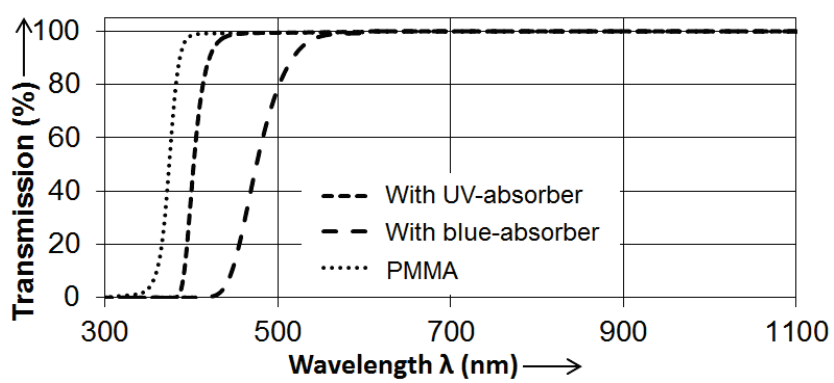

Fig. 3: Transmission spectrum of the investigated IOLmaterial. PMMA transmission spectrum is shown for comparison (sample thickness $\mathrm{d}=3 \mathrm{~mm}$ ).

\section{Experimental results}

\subsection{Ablation thresholds and Incubation}

To achieve high precision the laser matter interaction process must occur in the nonlinear regime via multiphoton absorption. In this case subsequent irradiation of polymers induces the incubation effect which is distinctly stronger as for linear absorption of light. By this term several effects are summarized like chemical decomposition of the polymer chains, creation of color centers [6] and formation of subsurface cracks. The first two can amplify linear absorption and the last one is reported to increase the local intensity by $n^{4}$ depending on the refractive index $n$ [7]. As these modifications directly influence the ablation threshold $F_{t h}$ which represents the specific fluence needed to reach ablation or as performed in this work to cause at least a visible modification of a material, threshold becomes strongly dependent on the number of laser pulses $N$ applied on the same area.

To quantify the incubation effect the ablation threshold is measured by the zero-damage method [8].

$$
D^{2}=2 w_{0}^{2} \ln \left(F_{0} / F_{t h}\right)
$$

with:

$$
\begin{array}{ll}
D: & \text { Diameter of ablated area } \\
2 w_{0}: & \text { Focal spot diameter } \\
F_{0}: & \text { Peak fluence }
\end{array}
$$

For this purpose, the polymer surface is ablated with different pulse energies and different numbers of pulses. The diameter of the modified or ablated area can be identified easily by light microscopy as shown in Fig 4. It should be mentioned here that only the diameter directly modified by the laser is considered for the measurements as brittle breaks at the edges misrepresent the results.

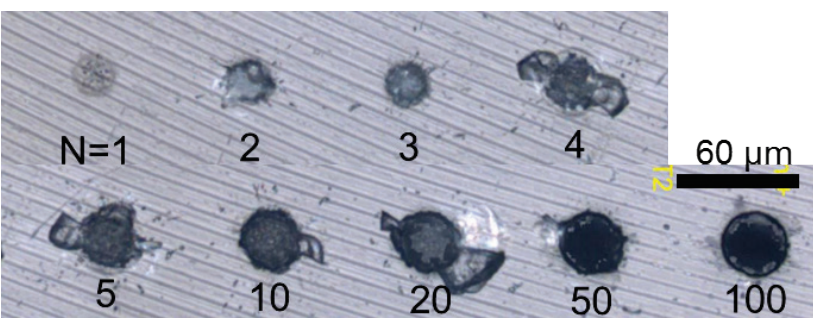

Fig. 4: Areas ablated with different numbers of laser pulses 
The ablation threshold $F_{\text {th }}$ and the respective focal spot radius $w o$ can be determined as described in [8]. First of all, the squared diameter of the ablated area is plotted versus the logarithm of the pulse energy. The gradient of the best fit straight line allows us to calculate the focal spot size wo. By identifying that size, the pulse energy depending peak fluence $F_{0}$ can be determined from the measured laser power. Finally, the squared diameter of the ablated area can be plotted semi-logarithmic as a function of the peak fluence. This was performed applying different numbers of pulses and is shown exemplary for different $N$ in Figure 5.

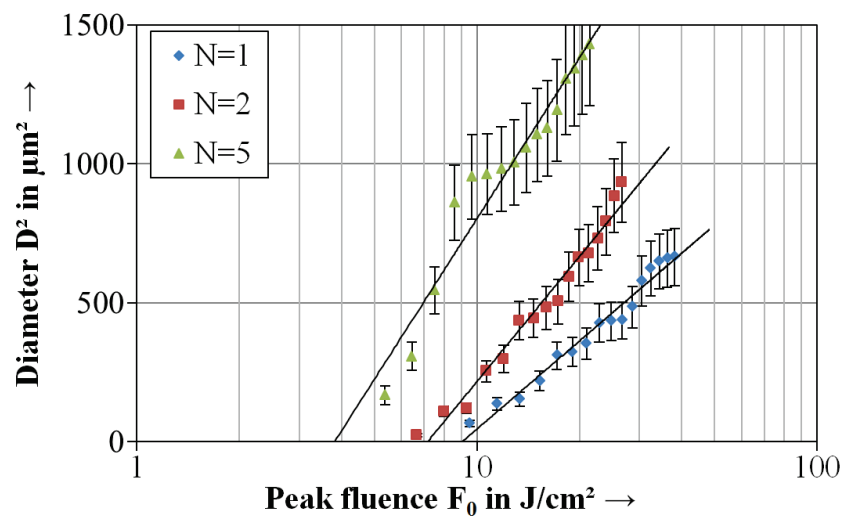

Fig. 5: Laser ablation threshold determination

Several empiric models have been proposed describing the evolution of incubation as shown in Fig. 6. The two most popular have been recommended in [6]:

$$
F_{t h}(N)=F_{t h, 1} N^{k-1}
$$

and in [7]:

$$
F_{t h}(N)=F_{t h, \infty}+\left(F_{t h, 1}-F_{t h, \infty}\right) e^{-k(N-1)}
$$

with:

$$
\begin{aligned}
& F_{\text {th }, 1}: \text { Ablation threshold at the first pulse } \\
& F_{\text {th, } \infty}: \text { Threshold after an infinite number of pulses } \\
& k: \quad \text { Incubation parameter }
\end{aligned}
$$

We apply a least square best fit on our experimental data for both models and this leads to values for the fit parameters listed below in Table 1 .

Table 1 Fit parameters of the incubation models

\begin{tabular}{ccc}
\hline Model: & Eq. (2) [7] & Eq. (3) [6] \\
\hline$F_{\text {th }, 1}$ & $9.20 \pm 1.54 \mathrm{~J} / \mathrm{cm}^{2}$ & $9.05 \pm 0.45 \mathrm{~J} / \mathrm{cm}^{2}$ \\
$F_{\text {th }, \infty}$ & - & $2.47 \pm 0.26 \mathrm{~J} / \mathrm{cm}^{2}$ \\
$k$ & $0.507 \pm 0.196$ & $0.386 \pm 0.062$ \\
\hline
\end{tabular}

For large numbers of pulses $(N>20)$ the fit line based on (2) starts to deviate strongly from the experimental data as it predicts an ablation threshold $F_{\mathrm{th}, \infty}=0 \mathrm{~J} / \mathrm{cm}^{2}$ for an infinite number of pulses. On the other hand the model based on (3) shows very good agreement to our experimental data and is used for further considerations.

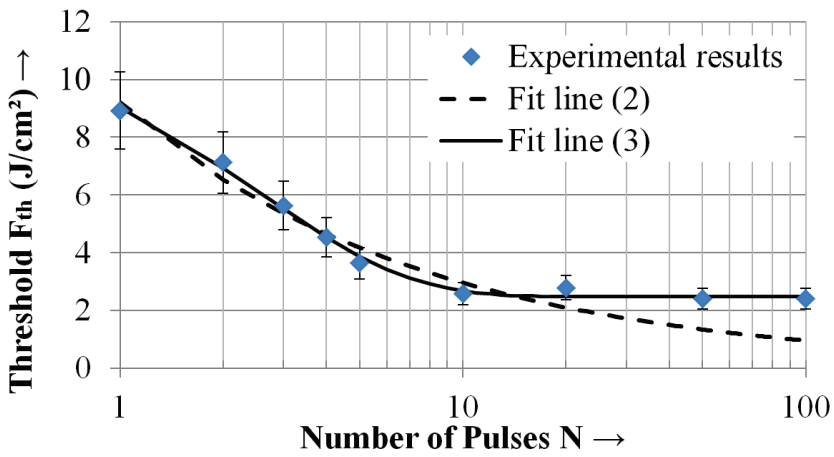

Fig. 6: Ablation threshold $F_{\text {th }}$ depending on the number of pulses $N$ applied on the same area

The model (2) expects a specific threshold for an infinite number of pulses, however, the value $F_{\mathrm{th}, \infty}$ is already reached above $N \approx 8$ pulses. This steady-state incubation of the irradiated area is caused by the equilibrium between ablation and modification of the material.

\subsection{Damage types}

Sample cuts are produced by linear movement of the focal spot of the laser beam over the sample surface. To identify suitable process parameters we perform a variation of pulse energy, pulse frequency, feed rate and the number of passes. Dividing the feed rate by the pulse frequency we can calculate the lateral distance between the pulses $d_{\mathrm{x}}$ which has more significance than the feed rate as it represents the spatial pulse distribution. Thereby, rather different results with a wide distribution of cutting quality can be achieved including proper results as shown in Fig. 7(c) but also several diverse damage types around the kerf which are described below.

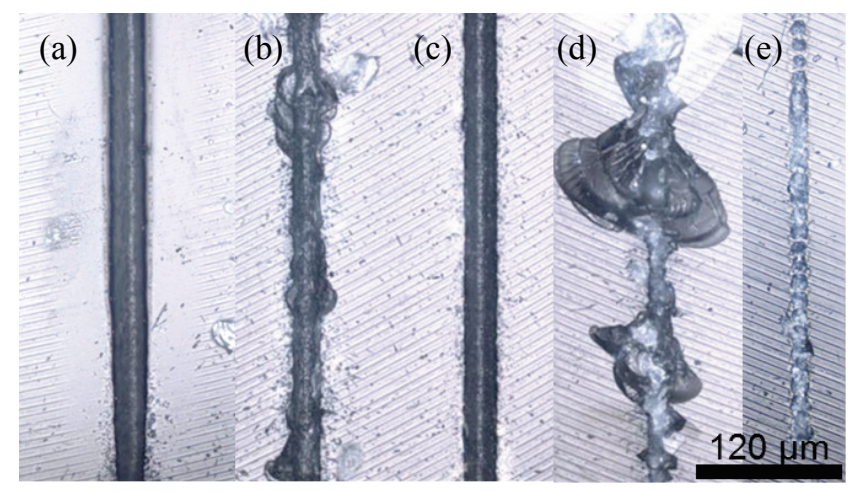

Fig. 7: Observed damage types due to cutting: (a) melting, (b) melting and breaking, (c) good result, (d) only brittle breaking, (e) incubated material with subsurface cracks)

At high pulse repetition rates and small pulse separation melting of the surrounding area is observed (Fig. 7(a) and red zone in Fig. 9). This can be explained by the accumulation of heat from one pulse to another as even for ultrafast laser processing a small amount of the energy is introduced as heat into the surrounding material. The time between subsequent pulses is short enough to prevent the heat from efficient dissipation and by adding more and more small amounts of heat from each pulse the temperature begins to 
increase. At a certain pulse frequency and pulse distance the temperature exceeds the glass transition temperature $\left(\mathrm{T}_{\mathrm{g}}=108^{\circ} \mathrm{C}\right)$ causing melting of the surface.

One could conclude that a possible solution preventing heat accumulation and maintaining processing speed would be to increase the pulse distance $d_{\mathrm{x}}$. However, as shown in Fig. 7(b) and (d) (grey area in Fig. 9) brittle breaking of the cut edges occurs above a certain pulse distance. Our hypothesis for this effect is an unfavorable distribution of subsurface cracks. These cracks are introduced by a pulse having a certain minimal distance to another and can be easily seen by light microscopy focusing with a high numerical aperture below the sample surface as shown in Fig. 8. These cracks act as a predetermined breaking route if distance is in a specific region $\left(2 \mu \mathrm{m}<d_{\mathrm{x}}<20 \mu \mathrm{m}\right)$ and cause the overlying material to chip off the surface.

If pulse distance is even higher only incubation at the surface is visible (Fig. 7(e) and corresponding to the blue parametric zone in Fig. 9) but subsurface cracks are also present. However, they do not link and cause no visible damage. If subsequent passes of the same area are executed with a high pulse distance brittle breaks at the surface occur too, for the same reason as mentioned before for the smaller distances.

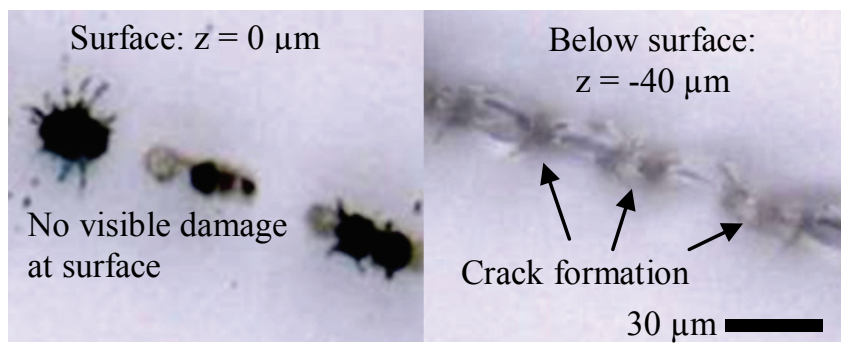

Fig. 8: Light microscopy images of the surface and $40 \mu \mathrm{m}$ below showing crack formation.

The very different results can be located within a process window visualizing the different damage types as well as suitable process parameters (Fig. 9).

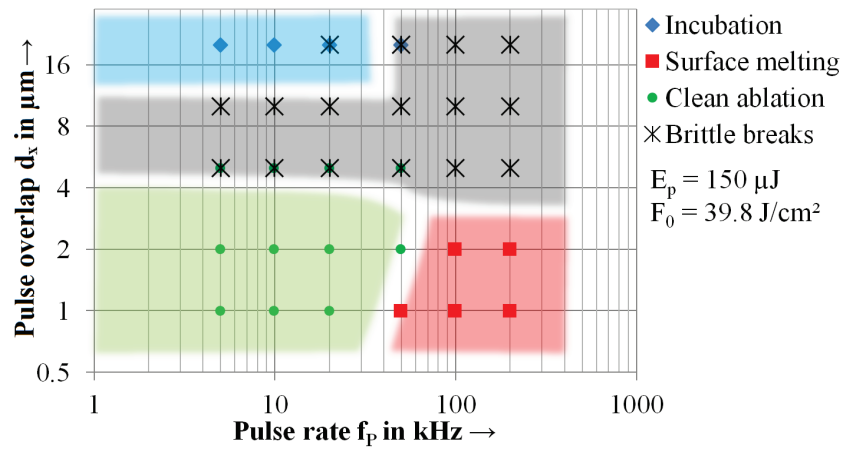

Fig. 9: Process window with localization of different types of damage

Best results concerning ablation quality are achieved at small pulse distances $d_{\mathrm{x}} \leq 2 \mu \mathrm{m}$. We propose that in this case, a crack which is introduced by the first pulse is completely decomposed by the following pulses. A suitable estimation for the number of pulses overlapping during linear movement can be found in [9]. In this article, Ma- chado et al. propose the following equation for $N$ depending on $d_{\mathrm{x}}$ and $w_{0}$ :

$$
N=1.25 w_{0} / d_{x}
$$

Using (4) we can recalculate $d_{\mathrm{x}} \leq 2 \mu \mathrm{m}$ from the parameters for clean ablation receiving $N \geq 9.7$. For this amount of pulses we conclude from our experiments concerning the ablation threshold that full incubation is reached and is apparently needed for proper ablation without damaging the surrounding area. It seems that introduced cracks which are found to be smaller than the ablated area above, and internal stress of a first pulse are decomposed by the following pulses and thereby cannot link to form a breaking route.

Comparing the investigated parameters shows that the most productive parameters for cutting with suitable quality are $E_{p}=150 \mu \mathrm{J}, \mathrm{d}_{\mathrm{x}}=2 \mu \mathrm{m}, \mathrm{f}_{\mathrm{p}}=50 \mathrm{kHz}$ with a resulting scan speed of $\mathrm{v}=100 \mathrm{~mm} / \mathrm{s}$. The ablation depth for this parameters is $\mathrm{h}=46 \mu \mathrm{m}$ for one passage.

\section{Conclusion and outlook}

Our study of the ablation behavior in laser cutting of intraocular lens polymers showed various factors strongly influence the cut quality as well as productivity. Among these, incubation plays a major role, as light-material interaction and absorption significantly changes from pulse to pulse. To achieve consistent results we figured out that a certain pulse overlap is needed to provide full incubation. By this approach, cracks do not form. If we try to scale up the process by increasing the pulse repetition rate, heat accumulation occurs for such small pulse distances. Therefore, a tradeoff can be found between processing speed and cut quality as shown in Fig. 10.

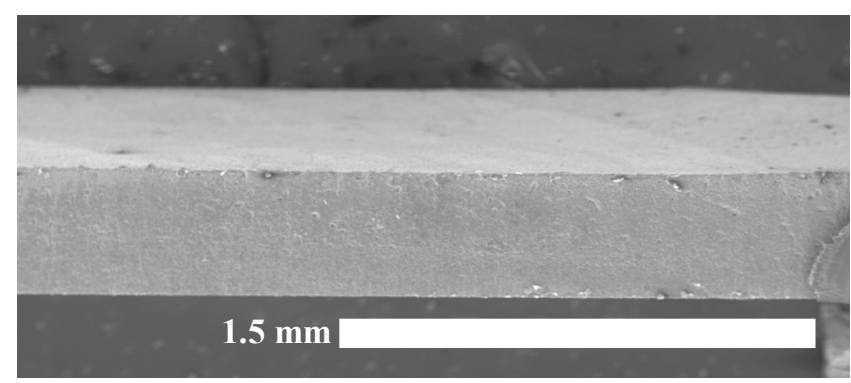

Fig. 10: SEM image of a cut surface

Based on our results we propose a manufacturing process for contours of intraocular lenses. This enables compared to the conventional techniques more flexibility due to the small kerf widths achievable by nonlinear absorption of the laser beam. The introduced heat is controllable at moderate pulse repetition rates and allows contactless cutting without any heat affected zones like known from conventional $\mathrm{CO}_{2}$-laser cutting of polymers.

\section{Acknowledgments}

We would like to thank the German Federal Ministry of Education and Research (BMBF) for funding the project "A-03: Erarbeitung technologischer Grundlagen für die spätere Entwicklung und Herstellung von optimierten 
phaken Intraokularlinsen - Teilprojekt 2: Oberflächenstrukturierung transparenter Biomaterialien mittels Laserstrahlung" (promotional reference: 13EX1011C) as well as our project partner the HumanOptics AG for providing the IOL-samples and the received support. The authors gratefully acknowledge funding of the Erlangen Graduate School in Advanced Optical Technologies (SAOT) by the German Research Foundation (DFG) in the framework of the German excellence initiative.

\section{References}

[1] S.D. McLeod, V. Portney and A. Ting: Brit. J. Ophthalmology. 87 (2003), pp. 1083-1085.

[2] M.C. Kraff, D. Sanders and H.L. Lieberman: J. Am. Intraocul. Implant. Soc. 93 (1983), pp. 301-305.

[3] F. Korte. S. Nolte, B.N. Chichkov, T. Bauer, G. Kamlage, T. Wagner, C. Fallnich and H. Welling: Appl. Phys. A, Vol. 69, No. 7 (1999), pp S7-S11

[4] N. Muhammad, D. Whitehead, A. Boor, W. Oppenlander, Z. Liu, L. Li: Appl. Phys. A, Vol. 106, No. 3 (2012), pp. 607-617.

[5] J. Heberle, F. Klämpfl, I. Alexeev and M. Schmidt: JLMN, Vol. 8, No. 1 (2013) pp. 51-55.

[6] Y. Jee, M. F. Becker, and R. M. Walser: J. Opt. Soc. Am. B 5(3) (1988), pp. 648-659.

[7] D. Ashkenasi, M. Lorenz, R. Stoian, A. Rosenfeld, Appl. Surf. Sci. 150 (1999), pp. 101-106

[8] J.M. Liu, Opt. Lett., Vol. 7, No. 5 (1982), pp.196-197

[9] L. M. Machado, R. E. Samad, W. de Rossi, and N. D. Vieira Junior: Opt. Expr. 20 (2012),pp. 4114-4123.

[10] N.C. Nayak, Y.C. Lam, C.Y. Yue, A. T. Sinha: J. Micromech. Microeng. 18 (2008) 095020 (7pp)

[11] http://www.contamac.com/Products/IntraocularLenses/CI26.aspx

(Received: July 23, 2013, Accepted: March 24, 2014) 